INPLASY

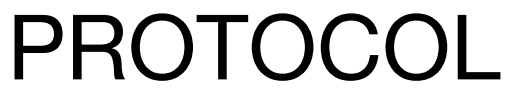

To cite: $\mathrm{Xu}$ et al. Wrist-Ankle Acupuncture: an additional therapy for Postoperative Multimodal Analgesia at Orthopaedic Surgery: A systematic review and metaanalysis. Inplasy protocol 2021100030. doi:

10.37766/inplasy2021.10.0030

Received: 09 October 2021

Published: 09 October 2021

Corresponding author: Ning Xu

xuning01@163.com

Author Affiliation: Weihai Central Hospital affiliated with Qingdao University School of Medicine.

Support: No financial support.

Review Stage at time of this submission: Formal screening of search results.

Conflicts of interest:

None declared.

\section{Wrist-Ankle Acupuncture: an additional therapy for Postoperative Multimodal Analgesia at Orthopaedic Surgery: A systematic review and meta-analysis}

\author{
$\mathrm{Xu}, \mathrm{N}^{1}$; Liu, L2; Rong, W3.
}

Review question / Objective: This meta-analysis aimed to analyze the efficacy and safety of wrist-ankle acupuncture performed as an additional therapy for postoperative multimodal analgesia at orthopaedic surgery.

Eligibility criteria: Study type: Randomized controlled trials(RCTs) or Cohort study; Participants: Orthopaedic surgical patients with no limitations of age, gender or surgical type; Interventions: Orthopaedic surgical patients who received wrist-ankle acupuncture as an additional therapy for postoperative analgesia (WAA group); Comparisons: Orthopaedic surgical patients who received other treatment for postoperative analgesia(C group); Outcome : different time points of visual analogue score (VAS) and consumption of PCIA analgesics; postoperative adverse events including nausea and vomiting, dizziness, drowsiness and gastrointestinal complications.

INPLASY registration number: This protocol was registered with the International Platform of Registered Systematic Review and Meta-Analysis Protocols (INPLASY) on 09 October 2021 and was last updated on 09 October 2021 (registration number INPLASY2021100030).

\section{INTRODUCTION}

Review question / Objective: This metaanalysis aimed to analyze the efficacy and safety of wrist-ankle acupuncture performed as an additional therapy for postoperative multimodal analgesia at orthopaedic surgery.

Rationale: To analyze the efficacy and safety of wrist-ankle acupuncture performed as an additional therapy for 
postoperative multimodal analgesia at orthopaedic surgery.

Condition being studied: Wrist-Ankle Acupuncture.

\section{METHODS}

Search strategy: We searched electronic databases such as Cochrane-library, PubMed, EMBASE, Web of Science, CNKI, SinoMed, Wanfang, and VIP to obtain randomized controlled trials(RCTs) or cohort studies that reported the details of wrist-ankle acupuncture performed as an additional therapy for postoperative multiple anesthesia at orthopaedic surgery before October 1st,2021.

Participant or population: Orthopaedic surgical patients with no limitations of age, gender or surgical type.

Intervention: Orthopaedic surgical patients who received wrist-ankle acupuncture as an additional therapy for postoperative analgesia.

Comparator: Orthopaedic surgical patients who received other treatment for postoperative analgesia.

Study designs to be included: Randomized controlled trials(RCTs) or Cohort study.

Eligibility criteria: Study type: Randomized controlled trials(RCTs) or Cohort study; Participants: Orthopaedic surgical patients with no limitations of age, gender or surgical type; Interventions: Orthopaedic surgical patients who received wrist-ankle acupuncture as an additional therapy for postoperative analgesia (WAA group); Comparisons: Orthopaedic surgical patients who received other treatment for postoperative analgesia(C group); Outcome : different time points of visual analogue score (VAS) and consumption of PCIA analgesics; postoperative adverse events including nausea and vomiting, dizziness, drowsiness and gastrointestinal complications.
Information sources: We searched the Cochrane-library, PubMed, EMBASE, Web of Science, SinoMed, CKNI, WanFang, and VIP databases, as well as dissertations and conference papers, from inception to October 1st,2021.

Main outcome(s): Different time points of visual analogue score (VAS) and consumption of PCIA analgesics; postoperative adverse events including nausea and vomiting, dizziness, drowsiness and gastrointestinal complications.

Quality assessment / Risk of bias analysis: The quality of the included studies was evaluated by two authors in light of the Cochrane Risk of Bias Assessment Tool. To perform certainty assessment for each outcome, we used the Grading Recommendations Assessment, Development and Evaluationg (GRADE) approach As a cumulative meta-analysis, trial sequential analysis(TSA) was performed to reduce the possibility of generating misleading evidence caused by random errors.

Strategy of data synthesis: pubMed strategy : "wrist-ankle"[Title/Abstract] "acupunctural"[Title/Abstract] OR "acupuncture"[MeSH Terms] OR "acupuncture"[Title/Abstract] OR "acupuncture therapy"[MeSH Terms] OR ("acupuncture"[Title/Abstract] AND "therapy"[Title/Abstract]) OR "acupuncture therapy"[Title/Abstract] OR "acupuncture s"[Title/Abstract] OR "acupunctured"[Title/ Abstract] OR "acupunctures"[Title/ Abstract] OR "acupuncturing"[Title/ Abstract] "postoperative period"[MeSH Terms] OR ("postoperative"[Title/Abstract] AND "period"[Title/Abstract]) OR "postoperative period"[Title/Abstract] OR "postop"[Title/Abstract] OR "postoperative"[Title/Abstract] OR "postoperatively"[Title/Abstract] OR "postoperatives "[ Title/Abstract] "multimodal imaging"[MeSH Terms] OR ("multimodal"[Title/Abstract] AND "imaging"[Title/Abstract]) OR "multimodal i m a ging " [ T it le / A b s t r a c t] OR "multimodality"[Title/Abstract] OR 
"multimodal"[Title/Abstract] OR

"multimodalities"[Title/Abstract]

"analgesia" [MeSH Terms] OR

"analgesia"[Title/Abstract] OR "analgesias"[Title/Abstract]) \#3 AND \#4 AND \#5 "orthopaedic"[Title/Abstract] OR "orthopedics"[MeSH Terms] OR

"orthopedics"[Title/Abstract] OR

"orthopedic"[Title/Abstract] OR

"orthopaedical"[Title/Abstract] OR

"orthopedical"[Title/Abstract] OR

"orthopaedics"[Title/Abstract].

Subgroup analysis: Based on the different time points for VAS assessment, we documented four time points: 12h, 24h, $48 \mathrm{~h}, 7 \mathrm{~d}$ after the surgery. In terms of consumption of PCIA analgesics, we used two time points: $24 \mathrm{~h}, 48 \mathrm{~h}$ after the surgery to analysis.In addition, we made postoperative adverse events divided into four aspects to perform a subgroup analysis including nausea and vomiting, dizziness, drowsiness and gastrointestinal complications.

Sensitivity analysis: We summerized a table to show the results of sensitivity analysis. According to the table ,we can obviously be aware how significant the heterogeneity is and where it came from.

Language: English.

Country(ies) involved: China.

Keywords: wrist-ankle Acupuncture, postoperative multimodal analgesia, orthopaedic surgery, meta-analysis.

Contributions of each author:

Author 1 - Ning Xu - The author drafted the manuscript.

Email: xuning01@163.com

Author 2 - Lulu Liu - The author provided statistical expertise.

Email: 15166118123@163.com

Author 3 - Wei Rong - The author contributed to the development of the selection criteria, and the risk of bias assessment strategy.

Email: 18306301858@163.com 